\title{
Genotypic Diversity of Beet Curly Top Virus Populations in the Western United States
}

\author{
Drake C. Stenger and Carrie L. McMahon
}

Department of Biological Sciences and Plant Molecular Biology Center, Northern Illinois University, DeKalb 60115. Accepted for publication 31 March 1997.

\section{ABSTRACT}

Stenger, D. C., and McMahon, C. L. 1997. Genotypic diversity of beet curly top virus populations in the western United States. Phytopathology 87:737-744.

The genotypic diversity of beet curly top virus (BCTV) present in the
western United States has been examined by the analysis of 58 field
isolates and eight laboratory or nursery isolates of the virus. Full-length
clones for each isolate have been characterized for genotype by restric-
tion endonuclease mapping. The results indicate that most of the geno-
types examined may be classified as variants of the CFH, Worland, or
Cal/Logan strains of BCTV. Two genotypes were recovered that appear
to share certain genotypic markers of both Worland and CFH strains.
Genotypic variants of the CFH and Worland strains and the two geno-
types sharing markers of both strains were recovered from field isolates
collected during 1994 and 1995 . In contrast, the Cal/Logan strain was
recovered only from isolates maintained in laboratories or nurseries.
Comparisons of restriction endonuclease maps of cloned BCTV genomes
revealed considerable variability both within and between strains. Al-

Beet curly top virus (BCTV) occurs in the western United States and has been recognized as a disease agent affecting sugar beet and other crops throughout the 20th century (2). As sugar beet cultivation became widespread in the western United States during the first quarter of the century, losses associated with BCTV threatened the viability of the industry. The introduction of sugar beet cultivars with improved tolerance or partial resistance to BCTV in the 1920s was largely responsible for the survival of the industry at that time. Nonetheless, BCTV continues to cause significant yield losses in sugar beet (and other crops) despite ongoing efforts to breed and select for cultivars that are able to produce acceptable yields in the presence of BCTV.

BCTV is a single-stranded DNA virus belonging to the family Geminiviridae, and it is the type member of subgroup II geminiviruses (5). BCTV may be distinguished from geminiviruses in subgroups I and III based on a monopartite genome of unique organization, transmission by the beet leafhopper, and an unusually wide host range among dicot species. BCTV may be distinguished from other subgroup II viruses, tomato pseudo-curly top virus (TPCTV) (3) and horseradish curly top virus (HrCTV) (13), based on biological properties (vector specificity, host range, or both) and genomic properties that indicate both TPCTV and HrCTV may be considered examples of naturally occurring recombinant viruses.

It is only in the past 10 years that BCTV has been characterized at the molecular level. Stanley et al. (17) first sequenced an infectious clone of a BCTV isolate originally collected near Modesto, CA, by Freitag in the 1930s. This isolate was maintained as a

Corresponding author: D. C. Stenger; E-mail address: dstenger@niu.edu

Publication no. P-1997-0425-01R

(C) 1997 The American Phytopathological Society though a total of 43 distinct genotypes of BCTV were identified, only 36 $(84 \%)$ were recovered from field isolates. Of 37 field isolates for which more than a single clone was recovered, $16(43 \%)$ contained more than a single genotype of one strain, whereas $4(11 \%)$ harbored mixed infections of the CFH and Worland strains. A phylogenetic analysis using 43 characters derived from restriction endonuclease mapping data supported the grouping of 41 genotypes into three taxa consistent with the three currently recognized strains of BCTV. The relationships of the two genotypes sharing genotypic markers of both the Worland and $\mathrm{CFH}$ strains to other BCTV genotypes was unresolved in the phylogenetic analysis. Based on the mild symptom phenotype of the isolates from which these two genotypes were recovered and the presence of Worland genotypic markers in portions of the genome containing both cis- and trans-acting elements determining replication specificity, these two genotypes were tentatively considered as variants of the Worland strain.

Additional keyword: geminivirus.

greenhouse culture by serial leafhopper transmission for nearly 50 years prior to cloning. Subsequently, three other laboratory-maintained isolates of BCTV (CFH, Worland, and Logan) that were collected from the field during the 1970s and 1980s have been cloned (21) and sequenced (10,18,23).

Biological and genomic characteristics have been used to classify sequenced isolates of BCTV into three strains. The California and Logan isolates of BCTV are indistinguishable in host range and symptom phenotype (21), share a number of common restriction endonuclease cleavage sites that are not conserved among the sequenced genomes of the Worland and CFH strains (21), and share an overall nucleotide sequence identity of $96.3 \%$ (23). Based on these similarities, it has been proposed that the California and Logan isolates of BCTV represent variants of a single strain, now designated as Cal/Logan (23). In contrast, the sequenced genomes of the $\mathrm{CFH}$ and Worland isolates were designated as representing two additional strains of BCTV sharing an overall nucleotide sequence identity of $80 \%$ with each other, and 79 to $83 \%$ with the Cal/Logan strain (23). Although the Worland strain causes severe symptoms on a variety of dicot species, Worland characteristically causes only mild symptoms on sugar beet cultivars that otherwise express severe symptoms when infected with $\mathrm{Cal} / \mathrm{Logan}$ or $\mathrm{CFH}$ (21). The CFH strain of BCTV has unique biological properties that distinguish it from the other two strains. In particular, the CFH strain has a shorter latent period on the experimental host Nicotiana benthamiana Domin. than do Logan (22) or Worland (19), is able to infect two ecotypes of Arabidopsis thaliana (L.) Heynh. that are resistant to Logan (14), and contains cis- and transacting elements conferring strain-specific DNA replication properties that are not separately interchangeable with the corresponding elements of the Logan isolate $(6,7)$. The inability of the Worland isolate to mobilize and amplify a Logan-derived defective-inter- 
fering DNA resident within a chromosome of transgenic $N$. benthamiana as a tandem repeat (19) suggests that the Worland strain also has strain-specific replication determinants that are distinct from the $\mathrm{Cal} /$ Logan strain. The behavior of Worland on A. thaliana ecotypes and the compatibility of heterologous replication elements among the $\mathrm{CFH}$ and Worland strains have yet to be examined.

Recently, efforts to assess the genotypic diversity of BCTV have been initiated. Four nursery isolates obtained from a BCTV population maintained and used by the sugar beet industry to assess cultivar response to infection were examined for genotypic complexity through the analysis of full-length clones recovered from each isolate (23). The results indicated that all four nursery isolates contained genotypic variants of the $\mathrm{Cal} / \mathrm{Logan}$ and $\mathrm{CFH}$ strains, and that one isolate also contained a genotypic variant of the Worland strain. In a study of genotypic diversity resident within a field population of the virus, six genotypic variants of the

TABLE 1. Previously characterized laboratory and nursery isolates of beet curly top virus

\begin{tabular}{|c|c|c|c|c|c|}
\hline Isolate & Origin & Representative clone & Strain & Genotype $^{\mathrm{a}}$ & Reference \\
\hline California & Modesto, CA & pBCTV028 & Cal/Logan & 40 & 17 \\
\hline Logan & Logan, UT & pLOGAN & $\mathrm{Cal} /$ Logan & 41 & 21 \\
\hline $\mathrm{CFH}$ & Unknown & $\mathrm{pCFH}$ & $\mathrm{CFH}$ & 1 & 21 \\
\hline Worland & Worland, WY & pWORLAND & Worland & 20 & 21 \\
\hline \multirow[t]{2}{*}{ BS94-1 } & Kimberly, ID & pBS94-1S-1 & Cal/Logan & 42 & 23 \\
\hline & & pBS94-1E-18 & $\mathrm{CFH}$ & 7 & 23 \\
\hline \multirow[t]{3}{*}{ BS94-3 } & Kimberly, ID & pBS94-3S-16 & Cal/Logan & 43 & 23 \\
\hline & & pBS94-3E-33 & $\mathrm{CFH}$ & 8 & 23 \\
\hline & & pBSDF94-3E-197 & Worland & 25 & 23 \\
\hline \multirow[t]{2}{*}{ BSDF94-1 } & Kimberly, ID & pBSDF94-1S-2 & $\mathrm{Cal} / \operatorname{Logan}$ & 43 & 23 \\
\hline & & pBSDF94-1E-78 & $\mathrm{CFH}$ & 9 & 23 \\
\hline \multirow[t]{2}{*}{ BSDF94-6 } & Kimberly, ID & pBSDF94-6S-1 & $\mathrm{Cal} / \operatorname{Logan}$ & 41 & 23 \\
\hline & & pBSDF94-6E-48 & $\mathrm{CFH}$ & 9 & 23 \\
\hline
\end{tabular}

a Genotype as defined by restriction endonuclease mapping as presented in Figure 2.

TABLE 2. Beet curly top virus field isolates and summary of cloning experiments

\begin{tabular}{|c|c|c|c|c|c|c|}
\hline Isolate & State & Locality & Representative clone & Number of clones & Strain & Genotype $^{a}$ \\
\hline NC94-2 & $\mathrm{CA}$ & Modesto & pNC94-2-175 & 3 & $\mathrm{CFH}$ & 15 \\
\hline CA95-1 & $\mathrm{CA}$ & Firebaugh & pCA95-1-28 & 1 & $\mathrm{CFH}$ & 6 \\
\hline CA95-2 & $\mathrm{CA}$ & Firebaugh & pCA95-2-52 & 2 & $\mathrm{CFH}$ & 6 \\
\hline CA95-4 & $\mathrm{CA}$ & Firebaugh & pCA95-4-10 & 1 & $\mathrm{CFH}$ & 6 \\
\hline CA95-9 & $\mathrm{CA}$ & Firebaugh & pCA95-9-181 & 1 & $\mathrm{CFH}$ & 16 \\
\hline \multirow[t]{2}{*}{ CO95-1 } & $\mathrm{CO}$ & Wiggins & pCO95-1-4 & 2 & Worland & 34 \\
\hline & & & pCO95-1-13 & 2 & Worland & 31 \\
\hline \multirow[t]{2}{*}{$\mathrm{CO} 95-3$} & $\mathrm{CO}$ & Wiggins & pCO95-3-27 & 3 & Worland ${ }^{\mathrm{b}}$ & 38 \\
\hline & & & pCO95-3-41 & 1 & Worland & 36 \\
\hline \multirow[t]{2}{*}{ CO95-6 } & $\mathrm{CO}$ & Ft. Morgan & pCO95-6-23 & 2 & Worland & 28 \\
\hline & & & pCO95-6-31 & 2 & Worland ${ }^{b}$ & 37 \\
\hline $\mathrm{CO} 95-7$ & $\mathrm{CO}$ & Ft. Morgan & pCO95-7-35 & 1 & Worland & 35 \\
\hline CO95-8 & $\mathrm{CO}$ & Ft. Morgan & pCO95-8-3 & 2 & Worland & 28 \\
\hline CO95-9 & $\mathrm{CO}$ & Ft. Morgan & pCO95-9-2 & 2 & Worland & 28 \\
\hline CO95-10 & $\mathrm{CO}$ & Ft. Morgan & pCO95-10-12 & 1 & Worland & 28 \\
\hline \multirow[t]{2}{*}{ ID95-1 } & ID & Grandview & pID95-1-23 & 2 & $\mathrm{CFH}$ & 7 \\
\hline & & & pID95-1-149 & 1 & $\mathrm{CFH}$ & 10 \\
\hline \multirow[t]{2}{*}{ ID95-2 } & ID & Grandview & pID95-2-1 & 1 & $\mathrm{CFH}$ & 11 \\
\hline & & & pID95-2-2 & 1 & $\mathrm{CFH}$ & 7 \\
\hline ID95-7 & ID & Nampa & pID95-7-41 & 1 & $\mathrm{CFH}$ & 11 \\
\hline ID95-8 & ID & Nampa & pID95-8-26 & 1 & $\mathrm{CFH}$ & 2 \\
\hline NM95-1 & NM & Artesia & pNM95-1-94 & 1 & $\mathrm{CFH}$ & 17 \\
\hline NM95-2 & NM & Artesia & pNM95-2-123 & 5 & $\mathrm{CFH}$ & 3 \\
\hline NM95-4 & NM & Artesia & pNM95-4-99 & 2 & $\mathrm{CFH}$ & 3 \\
\hline NM95-5 & NM & Artesia & pNM95-5-28 & 1 & Worland & 28 \\
\hline \multirow[t]{2}{*}{ NM95-6 ${ }^{c}$} & NM & Artesia & pNM95-6-49 & 1 & Worland & 29 \\
\hline & & & pNM95-6-60 & 1 & Worland & 30 \\
\hline \multirow[t]{2}{*}{ NM95-10 } & NM & Artesia & pNM95-10-7 & 1 & Worland & 25 \\
\hline & & & pNM95-10-21 & 3 & Worland & 31 \\
\hline \multirow[t]{2}{*}{ NM95-12c } & NM & Artesia & pNM95-12-3 & 1 & Worland & 32 \\
\hline & & & pNM95-12-18 & 1 & Worland & 33 \\
\hline OR95-3 & OR & Jameison & pOR95-3-103 & 2 & $\mathrm{CFH}$ & 7 \\
\hline OR95-6 & OR & Adrian & pOR95-6-74 & 1 & Worland & 23 \\
\hline \multirow[t]{2}{*}{ OR95-7 } & OR & Adrian & pOR95-7-54 & 1 & $\mathrm{CFH}$ & 7 \\
\hline & & & pOR95-7-69 & 2 & Worland & 23 \\
\hline OR95-8 & OR & Adrian & pOR95-8-17 & 2 & $\mathrm{CFH}$ & 7 \\
\hline \multirow[t]{3}{*}{ OR95-9 } & OR & Adrian & pOR95-9-31 & 2 & $\mathrm{CFH}$ & 16 \\
\hline & & & pOR95-9-47 & 1 & Worland & 25 \\
\hline & & & pOR95-9-67 & 1 & Worland & 31 \\
\hline OR95-10 & OR & Adrian & pOR95-10-106 & 1 & Worland & 23 \\
\hline
\end{tabular}

${ }^{a}$ Genotype as defined by restriction endonuclease mapping as described in Figure 2.

(continued on the next page)

b Shares more similarity to CFH than other genotypes of Worland.

c Pepper isolates; all others are from sugar beet.

${ }^{\mathrm{d}}$ Isolates and clones described previously in Stenger (20). 
CFH strain were recovered from 11 isolates of BCTV collected in the Texas panhandle during 1994 (20). Despite these previous efforts, information concerning the degree of diversity, complexity, and distribution of BCTV strains and genotypes throughout the western United States is inadequate. In this report, we present the results of studies in which isolates of BCTV collected from 16 localities in eight western states were examined for genotypic diversity, complexity, and strain composition.

\section{MATERIALS AND METHODS}

Sources of isolates and clones. Clones of BCTV derived from laboratory- or nursery-maintained isolates of BCTV are listed in Table 1. Isolates of BCTV collected from the field during 1994 and 1995 are listed in Table 2, and a map indicating the localities from which field isolates were obtained is presented in Figure 1. DNA clones derived from field isolates collected in 1994 from three Texas panhandle localities (Table 2) have been described previously (20); all other field isolates listed in Table 2 have not.

Cloning BCTV from field isolates. Total DNA was extracted from each field isolate as described (20). Total DNA extracts were digested with $E c o$ RI and analyzed by Southern hybridization using a full-length Logan riboprobe of virion sense (24). Double-stranded BCTV replicative form (RF) DNA was completely linearized in all cases such that a single genome-length linear molecule of 2.9 to $3.0 \mathrm{kbp}$ was detected in Southern hybridization following EcoRI digestion. Aliquots of EcoRI-digested DNA extracts $(50 \mu \mathrm{g})$ from each field isolate were size-fractionated in a $1 \%$ agarose gel, and size-selected DNA fragments $(2.7$ to $3.2 \mathrm{kbp})$ were recovered from the gel using Gene Clean II (Bio 101, La Jolla, CA). Size-

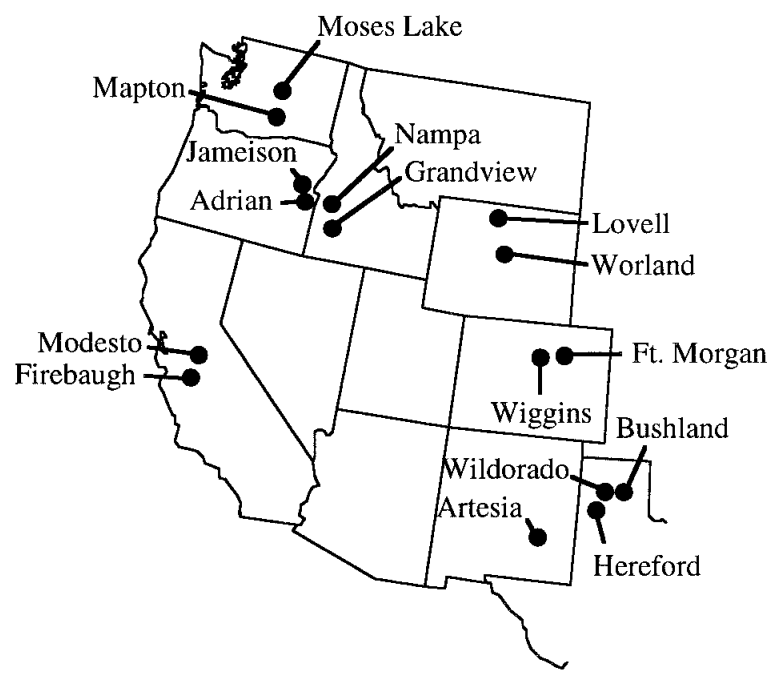

Fig. 1. Localities from which field isolates of beet curly top virus were collected in 1994 and 1995.

TABLE 2. (continued from the preceding page)

\begin{tabular}{|c|c|c|c|c|c|c|}
\hline Isolate & State & Locality & Representative clone & Number of clones & Strain & Genotype \\
\hline T94-1 ${ }^{\mathrm{d}}$ & $\mathrm{TX}$ & Bushland & pT94-1-25 & 5 & $\mathrm{CFH}$ & 4 \\
\hline \multirow[t]{2}{*}{ T94-4 ${ }^{\mathrm{d}}$} & $\mathrm{TX}$ & Bushland & pT94-4-59 & 6 & $\mathrm{CFH}$ & 3 \\
\hline & & & pT94-4-197 & 1 & $\mathrm{CFH}$ & 5 \\
\hline T94-6 ${ }^{\mathrm{d}}$ & $\mathrm{TX}$ & Bushland & pT94-6-9 & 5 & $\mathrm{CFH}$ & 1 \\
\hline T94-7 ${ }^{\mathrm{d}}$ & $\mathrm{TX}$ & Bushland & pT94-7-214 & 2 & $\mathrm{CFH}$ & 1 \\
\hline T94-12d & $\mathrm{TX}$ & Wildorado & pT94-12-87 & 3 & $\mathrm{CFH}$ & 1 \\
\hline T94-14 ${ }^{\mathrm{d}}$ & $\mathrm{TX}$ & Wildorado & pT94-14-157 & 1 & $\mathrm{CFH}$ & 6 \\
\hline T94-16 & $\mathrm{TX}$ & Wildorado & pT94-16-17 & 4 & $\mathrm{CFH}$ & 1 \\
\hline \multirow[t]{2}{*}{ T94-19d } & $\mathrm{TX}$ & Wildorado & pT94-19-109 & 4 & $\mathrm{CFH}$ & 1 \\
\hline & & & pT94-19-154 & 1 & $\mathrm{CFH}$ & 2 \\
\hline T94-23 ${ }^{d}$ & $\mathrm{TX}$ & Wildorado & pT94-23-7 & 1 & $\mathrm{CFH}$ & 2 \\
\hline T94-28d & $\mathrm{TX}$ & Hereford & pT94-28-124 & 1 & $\mathrm{CFH}$ & 3 \\
\hline T94-30d & $\mathrm{TX}$ & Hereford & pT94-30-188 & 1 & $\mathrm{CFH}$ & 3 \\
\hline Т95-1 & $\mathrm{TX}$ & Bushland & pT95-1-114 & 1 & $\mathrm{CFH}$ & 18 \\
\hline \multirow[t]{2}{*}{ T95-2 } & $\mathrm{TX}$ & Bushland & pT95-2-12 & 1 & $\mathrm{CFH}$ & 16 \\
\hline & & & pT95-2-49 & 1 & $\mathrm{CFH}$ & 18 \\
\hline T95-3 & $\mathrm{TX}$ & Bushland & pT95-3-95 & 1 & $\mathrm{CFH}$ & 18 \\
\hline T95-4 & $\mathrm{TX}$ & Bushland & pT95-4-13 & 4 & $\mathrm{CFH}$ & 18 \\
\hline \multirow[t]{2}{*}{ WA95-1 } & WA & Mapton & pWA95-1-71 & 5 & Worland & 21 \\
\hline & & & pWA95-1-17 & 3 & Worland & 22 \\
\hline \multirow[t]{3}{*}{ WA95-2 } & WA & Mapton & pWA95-2-47 & 4 & Worland & 23 \\
\hline & & & pWA95-2-192 & 1 & Worland & 24 \\
\hline & & & pWA95-2-221 & 1 & Worland & 25 \\
\hline WA95-3 & WA & Mapton & pWA95-3-106 & 4 & Worland & 26 \\
\hline ML95-1 & WA & Moses Lake & pML95-1-64 & 1 & Worland & 27 \\
\hline WD95-3 & WY & Worland & pWD95-3-75 & 1 & $\mathrm{CFH}$ & 12 \\
\hline \multirow[t]{2}{*}{ WD95-5 } & WY & Worland & pWD95-5-166 & 3 & $\mathrm{CFH}$ & 6 \\
\hline & & & pWD95-5-115 & 1 & $\mathrm{CFH}$ & 14 \\
\hline WD95-6 & WY & Worland & pWD95-6-14 & 3 & $\mathrm{CFH}$ & 6 \\
\hline WD95-8 & WY & Worland & pWD95-8-3 & 1 & $\mathrm{CFH}$ & 6 \\
\hline \multirow[t]{2}{*}{ WD95-18 } & WY & Worland & pWD95-18-14 & 1 & $\mathrm{CFH}$ & 13 \\
\hline & & & pWD95-18-46 & 1 & $\mathrm{CFH}$ & 12 \\
\hline WD95-20 & WY & Worland & pWD95-20-54 & 1 & $\mathrm{CFH}$ & 12 \\
\hline \multirow[t]{3}{*}{ WY95-1 } & WY & Lovell & pWY95-1-93 & 1 & Worland & 28 \\
\hline & & & pWY95-1-95 & 1 & Worland & 23 \\
\hline & & & pWY95-1-101 & 2 & Worland & 31 \\
\hline \multirow[t]{3}{*}{ WY95-2 } & WY & Lovell & pWY95-2-17 & 1 & $\mathrm{CFH}$ & 6 \\
\hline & & & pWY95-2-6 & 2 & Worland & 25 \\
\hline & & & pWY95-2-39 & 1 & Worland & 23 \\
\hline WY95-3 & WY & Lovell & pWY95-3-62 & 1 & Worland & 23 \\
\hline \multirow[t]{4}{*}{ WY95-5 } & WY & Worland & pWY95-5-8 & 1 & Worland & 25 \\
\hline & & & pWY95-5-26 & 1 & Worland & 39 \\
\hline & & & pWY95-5-35 & 1 & $\mathrm{CFH}$ & 12 \\
\hline & & & pWY95-5-98 & 1 & $\mathrm{CFH}$ & 19 \\
\hline
\end{tabular}


selected DNA recovered for each isolate was ligated to EcoRIdigested pUC8 and used to transform Escherichia coli strain DH5 $\alpha$. Bacterial clones bearing recombinant plasmids were identified by color selection in the presence of X-gal (Gibco BRL, Gaithersburg, MD) and screened for BCTV inserts by colony hybridization using a full-length Logan riboprobe as described previously (20).

Characterization of cloned BCTV genomes. Plasmid DNA was purified from clones that tested positive for BCTV DNA inserts by colony hybridization. Restriction endonuclease maps were constructed for each clone using the following enzymes: ApaI, BamHI, BstXI, Csp45I, EcoRI, HindIII, KpnI, PvuII, SacI, SalI, ScaI, SnaBI, SpeI, XbaI, and XhoI. Strain affiliation and specific genotypes were assigned to each clone based upon restriction endonuclease maps. A binary character state code was assigned to each genotype, such that there were 43 characters based on restriction endonuclease data $(0=$ absence of a site, $1=$ presence of a site). The binary character state code data were used to estimate phylogenetic relationships among the BCTV genotypes by parsimony performed with the PAUP 3.1.1 program (Illinois Natural History Survey, Champaign, IL) using a heuristic search with the branch-swapping option in effect. Phylogenetic trees were constructed using bootstrap resampling (1,000 replicates) in which only nodes present in a majority of trees examined were retained in the majority-rule consensus cladogram.

\section{$\mathrm{CFH}$}
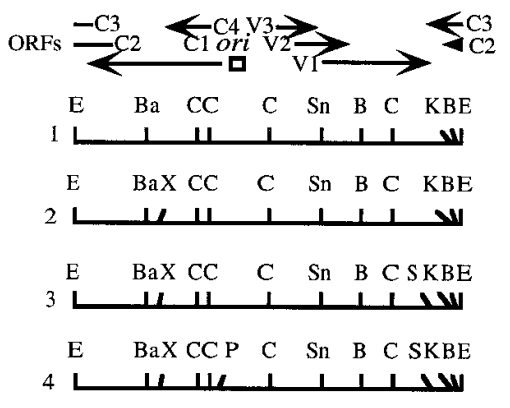

E PBaX CC C Sn B C SKBE

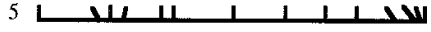

E $\quad$ Ba $\mathrm{CC} \quad \mathrm{K}$ Sn $\quad$ B $\quad \mathrm{C} \quad \mathrm{KBE}$

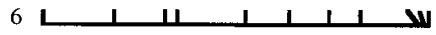

E BaXCC A Sn B CSpKBE

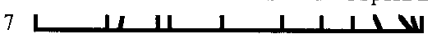

E $\quad$ Ba $\quad$ CC A $\quad$ AnspB C $\quad$ KBE

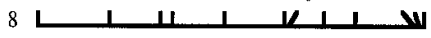

E $\quad$ Ba $\quad$ CC $\quad$ Sn $\quad$ B $\quad$ C $\quad$ KBE

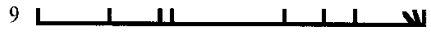

E $\quad$ BaX CC Sn B CSpKBE

10 W

E $\quad$ Ba $\quad$ CC $\quad$ CK $\quad$ Sn $\quad$ B $\quad$ CSpKBE

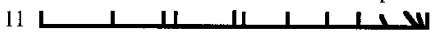

E $\quad$ Ba $\quad$ CC $\quad$ CK $\quad$ Sn $\quad$ B C $\quad$ KBE

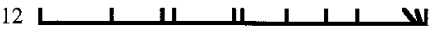

E $\quad \mathrm{Ba} \quad \mathrm{C} \mathrm{C}$ CK Sn B C KBE

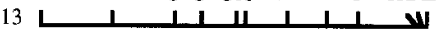

$\begin{array}{lllllll}\text { E } & \text { Ba } & \text { CC } & \text { Sn } & \text { B } & \text { C } & \text { KBE }\end{array}$

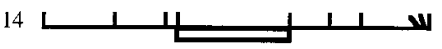

E Ba CC CK SnB B C KBE

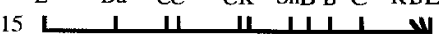

$\begin{array}{lllllll}\text { E } & \mathrm{Ba} & \mathrm{CC} & \mathrm{CK} & \mathrm{B} \mathrm{B} & \mathrm{C} & \mathrm{KBE}\end{array}$

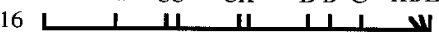

E B BaX CC C Sn B C SKBE

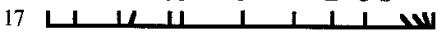

E BaX CC C Sn B CSps KBE

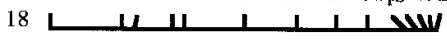

E $\quad \mathrm{Ba} \quad \mathrm{C} \quad \mathrm{CK}$ Sn B $\mathrm{C} \quad \mathrm{KBE}$

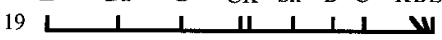

Worland

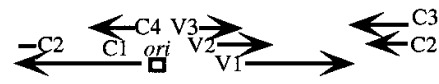

E Xb P S XbSpSn Sn B CSpSBaE

20 L

E Xb P S SpCSnSn B C Sp E

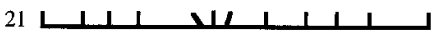

E Xb P S SpCSnCSn B C Sp E

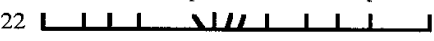

E XbP S SpCSn SnH B C Sp E

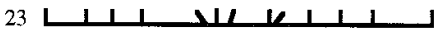

$\begin{array}{llllllllllll} & \mathrm{Xb} & \mathrm{S} & \mathrm{Sp} S \mathrm{Sn} & \mathrm{B} & \mathrm{C} & \mathrm{Sp} & \mathrm{E}\end{array}$

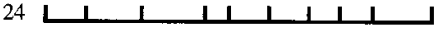

E Xb P S SpSn Sn B C Sp E

25 ل 25

E XbP S SpC Sn B C Sp E

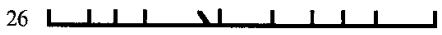

E Xb P S SpCSnBaSn B C Sp E

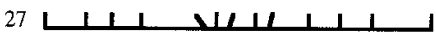

E XbP S Sp Sn B C Sp S E

ال

E XbP S Sn Sn B C SpS E

29 لــلـL

E Xb P SpSn Sn B C Sp E

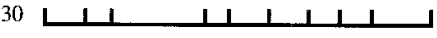

E XbP S SpSn Sn B C SpS E

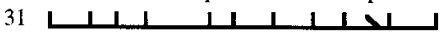

E Xb P S CSn Sn B C SpS E

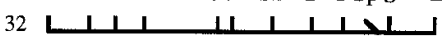

EPXb P S CSn Sn B CSpS E

33 ل

E XbP S SpSn Sn C SpS E

34 ل 34

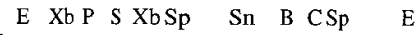

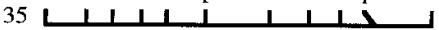

E XbP S SpA Sn B CSpS E

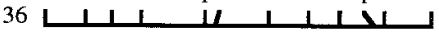

37 E $\quad$ P $\quad S \quad$ SpSn Sn B CSpS KBE

E XbP S Sp Sn HBCCXbKBE

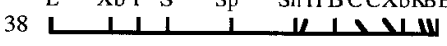

E Xb Sp Sn Sn B C Sp E

39 L

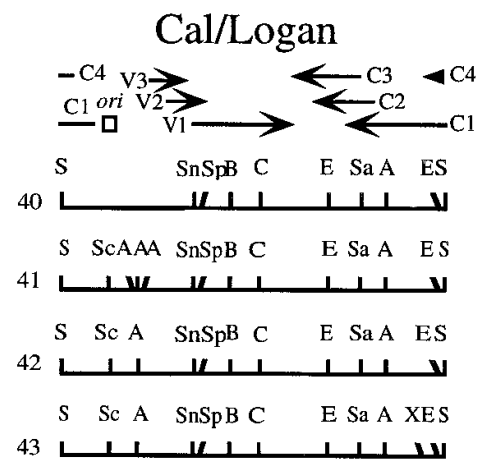

Fig. 2. Genotypic variants of beet curly top virus (BCTV) strains CFH, Worland, and Cal/Logan. Presented are restriction endonuclease maps determined for cloned variants of BCTV recovered from field, nursery, and laboratory isolates as listed in Tables 1 and 2 . Arrows denote locations and polarity of open reading frames (ORFs) C1-C4 and V1-V3 that are conserved among sequenced BCTV genomes. The location of cis-acting sequences of the origin of DNA replication (ori) is denoted by a box. Distinct genotypes are labeled by the number at the left of each restriction map. Abbreviations for restriction enzymes are A $=$ Apa $\mathrm{I}$, $\mathrm{Ba}=$ BamHI, B = BstXI, C = Csp45I, E = EcoRI, H = HindIII, K = KpnI, P = PvuII, Sa = SacI, S = SalI, Sc = ScaI, Sn = SnaBI, Sp = Spe I, Xb = XbaI, and X = $X$ hoI. The rectangle associated with genotype 14 denotes a region in which a deletion of 50 to 100 nucleotides appears to have occurred. 


\section{RESULTS}

Full-length BCTV DNA clones derived from 47 field isolates collected during this study (Table 2), 11 field isolates previously examined (Table 2) (20), and eight laboratory or nursery isolates previously examined (Table 1) $(17,21,23)$ were characterized for genotype by restriction endonuclease mapping (Fig. 2). Of a total of 43 genotypes defined among clones derived from field, laboratory, or nursery isolates of the virus, alignments of restriction endonuclease maps facilitated the classification of 41 geno-

TABLE 3. Genotypic complexity of beet curly top virus field isolates ${ }^{\mathrm{a}}$

\begin{tabular}{llccc}
\hline $\begin{array}{l}\text { Clones/ } \\
\text { isolate }\end{array}$ & \multicolumn{1}{c}{ Complexity } & $\begin{array}{c}\text { No. of } \\
\text { isolates }\end{array}$ & $\begin{array}{c}\% \text { of isolates } \\
(\text { all })\end{array}$ & $\begin{array}{c}\% \text { of isolates } \\
(\text { clones }>1)^{\mathrm{b}}\end{array}$ \\
\hline 1 & ND $^{\mathrm{c}}$ & 21 & 36 & $\mathrm{ND}^{\mathrm{c}}$ \\
$>1$ & 1 genotype & 17 & 29 & 46 \\
$>1$ & $>1$ genotype, 1 strain & 16 & 28 & 43 \\
$>1$ & $>1$ genotype, $>1$ strain & 4 & 7 & 11 \\
\hline
\end{tabular}

a Based on 58 isolates described in Table 2.

${ }^{\mathrm{b}} n=37$ isolates.

c $\mathrm{ND}=$ not determined.

TABLE 4. Occurrence of beet curly top virus genotypes in field isolates ${ }^{\mathrm{a}}$

\begin{tabular}{|c|c|c|c|c|c|}
\hline \multirow[b]{2}{*}{ Genotype } & \multirow[b]{2}{*}{ Strain } & \multicolumn{4}{|c|}{ Frequency } \\
\hline & & Isolates & Clones & Localities & States \\
\hline 1 & $\mathrm{CFH}$ & 5 & 18 & 2 & 1 \\
\hline 2 & $\mathrm{CFH}$ & 3 & 3 & 2 & 2 \\
\hline 3 & $\mathrm{CFH}$ & 5 & 15 & 3 & 2 \\
\hline 4 & $\mathrm{CFH}$ & 1 & 5 & 1 & 1 \\
\hline 5 & $\mathrm{CFH}$ & 1 & 1 & 1 & 1 \\
\hline 6 & $\mathrm{CFH}$ & 8 & 13 & 4 & 3 \\
\hline 7 & $\mathrm{CFH}$ & 4 & 7 & 3 & 2 \\
\hline 8 & $\mathrm{CFH}$ & 0 & 0 & 0 & 0 \\
\hline 9 & $\mathrm{CFH}$ & 0 & 0 & 0 & 0 \\
\hline 10 & $\mathrm{CFH}$ & 2 & 2 & 1 & 1 \\
\hline 11 & $\mathrm{CFH}$ & 2 & 2 & 2 & 1 \\
\hline 12 & $\mathrm{CFH}$ & 4 & 4 & 2 & 1 \\
\hline 13 & $\mathrm{CFH}$ & 1 & 1 & 1 & 1 \\
\hline 14 & $\mathrm{CFH}$ & 1 & 1 & 1 & 1 \\
\hline 15 & $\mathrm{CFH}$ & 1 & 3 & 1 & 1 \\
\hline 16 & $\mathrm{CFH}$ & 3 & 4 & 3 & 3 \\
\hline 17 & $\mathrm{CFH}$ & 1 & 1 & 1 & 1 \\
\hline 18 & $\mathrm{CFH}$ & 4 & 7 & 1 & 1 \\
\hline 19 & $\mathrm{CFH}$ & 1 & 1 & 1 & 1 \\
\hline 20 & Worland & 0 & 0 & 0 & 0 \\
\hline 21 & Worland & 1 & 5 & 1 & 1 \\
\hline 22 & Worland & 1 & 3 & 1 & 1 \\
\hline 23 & Worland & 7 & 11 & 3 & 3 \\
\hline 24 & Worland & 1 & 1 & 1 & 1 \\
\hline 25 & Worland & 5 & 6 & 5 & 4 \\
\hline 26 & Worland & 1 & 4 & 1 & 1 \\
\hline 27 & Worland & 1 & 1 & 1 & 1 \\
\hline 28 & Worland & 6 & 9 & 3 & 3 \\
\hline 29 & Worland & 1 & 1 & 1 & 1 \\
\hline 30 & Worland & 1 & 1 & 1 & 1 \\
\hline 31 & Worland & 4 & 8 & 4 & 4 \\
\hline 32 & Worland & 1 & 1 & 1 & 1 \\
\hline 33 & Worland & 1 & 1 & 1 & 1 \\
\hline 34 & Worland & 1 & 2 & 1 & 1 \\
\hline 35 & Worland & 1 & 1 & 1 & 1 \\
\hline 36 & Worland & 1 & 1 & 1 & 1 \\
\hline 37 & Worland & 1 & 2 & 1 & 1 \\
\hline 38 & Worland & 1 & 3 & 1 & 1 \\
\hline 39 & Worland & 1 & 1 & 1 & 1 \\
\hline 40 & Cal/Logan & 0 & 0 & 0 & 0 \\
\hline 41 & Cal/Logan & 0 & 0 & 0 & 0 \\
\hline 42 & $\mathrm{Cal} / \operatorname{Logan}$ & 0 & 0 & 0 & 0 \\
\hline 43 & Cal/Logan & 0 & 0 & 0 & 0 \\
\hline Mean $^{b}$ & & 1.95 & 3.49 & 1.40 & 1.23 \\
\hline
\end{tabular}

a Isolates described in Table 2.

b Per genotype. types as minor variants of the $\mathrm{CFH}$ (genotypes 1 to 19), Worland (genotypes 20 to 36, and 39), or Cal/Logan (genotypes 40 to 43) strains. BCTV genotypes 37 and 38 also were tentatively classified as Worland variants. It was noted, however, that these two genotypes contained more restriction sites in common with some variants of the CFH strain than did other variants of the Worland strain.

The genotypic complexity of 37 field isolates for which more than a single clone was obtained is presented in Table 3 . Of these 37 field isolates, approximately half yielded clones of a single genotype per isolate. The remaining isolates for which more than a single clone was examined most often contained more than one minor variant of the same strain, although four isolates harbored mixed infections of the Worland and CFH strains (Table 3). Although 43 genotypes of BCTV have been identified, only 36 were recovered from field isolates (Table 4). Surprisingly, none of the four known variants of the Cal/Logan strain were recovered from the field isolates examined. In contrast, genotypic variants of the $\mathrm{CFH}$ and Worland strains were common and widely distributed in the western United States. Despite the widespread occurrence of the $\mathrm{CFH}$ and Worland strains, individual genotypic variants were often recovered from only one or two localities within a single state (Table 4). Of a total of 150 clones examined from 58 field

TABLE 5. Occurrence of restriction endonuclease sites among genotypes of beet curly top virus

\begin{tabular}{|c|c|c|c|c|c|}
\hline \multirow[b]{2}{*}{$\begin{array}{l}\text { Site } \\
\text { no. }^{\text {a }}\end{array}$} & \multirow[b]{2}{*}{ Enzyme } & \multicolumn{4}{|c|}{ Frequency $(\%)$} \\
\hline & & $\begin{array}{c}\text { CFH } \\
(n=19)\end{array}$ & $\begin{array}{l}\text { Worland } \\
(n=20) \\
\end{array}$ & $\begin{array}{c}\mathrm{Cal} / \text { Logan } \\
(n=4)\end{array}$ & $\begin{array}{c}\text { All } \\
(n=43)\end{array}$ \\
\hline 1 & EcoRI & $19(100 \%)$ & $2(10 \%)$ & $0(0 \%)$ & $21(49 \%)$ \\
\hline 2 & BstXI & $1(5 \%)$ & $0(0 \%)$ & $0(0 \%)$ & $1(2 \%)$ \\
\hline 3 & $P v u \mathrm{II}$ & $0(0 \%)$ & $1(5 \%)$ & $0(0 \%)$ & $1(2 \%)$ \\
\hline 4 & BamHI & $19(100 \%)$ & $0(0 \%)$ & $0(0 \%)$ & $19(44 \%)$ \\
\hline 5 & XhoI & $8(42 \%)$ & $0(0 \%)$ & $1(25 \%)$ & $9(21 \%)$ \\
\hline 6 & Csp45I & $17(89 \%)$ & $0(0 \%)$ & $0(0 \%)$ & $17(40 \%)$ \\
\hline 7 & Csp45I & $19(100 \%)$ & $0(0 \%)$ & $0(0 \%)$ & $19(44 \%)$ \\
\hline 8 & Csp 45I & $1(5 \%)$ & $0(0 \%)$ & $0(0 \%)$ & $1(2 \%)$ \\
\hline 9 & ApaI & $2(11 \%)$ & $1(5 \%)$ & $3(75 \%)$ & $6(14 \%)$ \\
\hline 10 & Csp45I & $13(68 \%)$ & $7(35 \%)$ & $0(0 \%)$ & $20(47 \%)$ \\
\hline 11 & Кри I & $7(37 \%)$ & $0(0 \%)$ & $0(0 \%)$ & $7(16 \%)$ \\
\hline 12 & SnaBI & $18(95 \%)$ & $20(100 \%)$ & $4(100 \%)$ & $42(98 \%)$ \\
\hline 13 & SpeI & $1(5 \%)$ & $0(0 \%)$ & $4(100 \%)$ & $5(12 \%)$ \\
\hline 14 & BstXI & $2(11 \%)$ & $0(0 \%)$ & $0(0 \%)$ & $2(5 \%)$ \\
\hline 15 & BstXI & $19(100 \%)$ & $19(95 \%)$ & $4(100 \%)$ & $42(98 \%)$ \\
\hline 16 & $\operatorname{Csp} 45 \mathrm{I}$ & $19(100 \%)$ & $20(100 \%)$ & $4(100 \%)$ & $43(100 \%)$ \\
\hline 17 & SpeI & $4(21 \%)$ & $19(95 \%)$ & $0(0 \%)$ & $23(53 \%)$ \\
\hline 18 & SalI & $5(26 \%)$ & $9(45 \%)$ & $0(0 \%)$ & $14(33 \%)$ \\
\hline 19 & KpnI & $19(100 \%)$ & $2(10 \%)$ & $0(0 \%)$ & $21(49 \%)$ \\
\hline 20 & BstXI & $19(100 \%)$ & $2(10 \%)$ & $0(0 \%)$ & $21(49 \%)$ \\
\hline 21 & EcoRI & $0(0 \%)$ & $18(90 \%)$ & $0(0 \%)$ & $18(42 \%)$ \\
\hline 22 & $P v u \mathrm{II}$ & $0(0 \%)$ & $1(5 \%)$ & $0(0 \%)$ & $1(2 \%)$ \\
\hline 23 & $X b a \mathrm{I}$ & $0(0 \%)$ & $19(95 \%)$ & $0(0 \%)$ & $19(44 \%)$ \\
\hline 24 & $P v u \mathrm{II}$ & $0(0 \%)$ & $18(90 \%)$ & $0(0 \%)$ & $18(42 \%)$ \\
\hline 25 & SalI & $0(0 \%)$ & $18(90 \%)$ & $4(100 \%)$ & $22(51 \%)$ \\
\hline 26 & $X b a \mathrm{I}$ & $0(0 \%)$ & $2(10 \%)$ & $0(0 \%)$ & $2(5 \%)$ \\
\hline 27 & SpeI & $0(0 \%)$ & $17(85 \%)$ & $0(0 \%)$ & $17(40 \%)$ \\
\hline 28 & SnaBI & $0(0 \%)$ & $15(75 \%)$ & $0(0 \%)$ & $15(35 \%)$ \\
\hline 29 & Csp45I & $0(0 \%)$ & $1(5 \%)$ & $0(0 \%)$ & $1(2 \%)$ \\
\hline 30 & BamHI & $0(0 \%)$ & $1(5 \%)$ & $0(0 \%)$ & $1(2 \%)$ \\
\hline 31 & HindIII & $0(0 \%)$ & $2(10 \%)$ & $0(0 \%)$ & $2(5 \%)$ \\
\hline 32 & Csp 45I & $0(0 \%)$ & $1(5 \%)$ & $0(0 \%)$ & $1(2 \%)$ \\
\hline 33 & $X b a \mathrm{I}$ & $0(0 \%)$ & $1(5 \%)$ & $0(0 \%)$ & $1(2 \%)$ \\
\hline 34 & BamHI & $0(0 \%)$ & $1(5 \%)$ & $0(0 \%)$ & $1(2 \%)$ \\
\hline 35 & ScaI & $0(0 \%)$ & $0(0 \%)$ & $3(75 \%)$ & $3(7 \%)$ \\
\hline 36 & ApaI & $0(0 \%)$ & $0(0 \%)$ & $1(25 \%)$ & $1(2 \%)$ \\
\hline 37 & ApaI & $0(0 \%)$ & $0(0 \%)$ & $1(25 \%)$ & $1(2 \%)$ \\
\hline 38 & EcoRI & $0(0 \%)$ & $0(0 \%)$ & $4(100 \%)$ & $4(9 \%)$ \\
\hline 39 & SacI & $0(0 \%)$ & $0(0 \%)$ & $4(100 \%)$ & $4(9 \%)$ \\
\hline 40 & ApaI & $0(0 \%)$ & $0(0 \%)$ & $4(100 \%)$ & $4(9 \%)$ \\
\hline 41 & EcoRI & $0(0 \%)$ & $0(0 \%)$ & $4(100 \%)$ & $4(9 \%)$ \\
\hline 42 & PvuII & $1(5 \%)$ & $0(0 \%)$ & $0(0 \%)$ & $1(2 \%)$ \\
\hline
\end{tabular}

${ }^{\text {a }}$ Site numbers as assigned in Figure 3. 
isolates, each of the 43 genotypes was represented in an average of slightly fewer than two isolates per genotype, and each was recovered from an average of 1.4 of 16 localities in an average of 1.23 of eight states surveyed. Just over half (22 of 43 ) of the genotypes were recovered from only a single isolate each. Nonetheless, several genotypes of the CFH and Worland strains were encountered more frequently in the field survey. In particular, Worland genotypes $23,25,28$, and 31 were recovered from localities in three or four states, whereas CFH genotypes 6 and 16 were recovered from localities in three states (Table 4).

Composite restriction endonuclease maps were constructed for each of the three strains of BCTV and are presented in Figure 3. From these composite maps, it is apparent that certain restriction endonuclease sites are conserved among all characterized variants of a single strain, while other restriction endonuclease sites are variable and contribute to polymorphism within a strain. Of 21 restriction endonuclease sites occurring among 19 genotypic variants of the CFH strain, one-third were conserved among all of the $\mathrm{CFH}$ genotypes examined. Only two of 24 restriction endonuclease sites were conserved among all 20 genotypic variants of the Worland strain. Although the Cal/Logan strain appears to have more conserved than variable restriction endonuclease sites, this result may reflect the small sample size (four genotypes from six isolates) for which data are available. Of 42 endonuclease restriction sites mapped among the genotypic variants and a region in which a deletion has occurred within genotype 14, all but one of these characters exhibited polymorphism (Table 5, Fig. 3). Only the Csp45I site (designated no. 16) present within the coat protein open reading frame V1 was conserved among all 43 genotypes examined. A majority (30 of 42) of the restriction endonuclease sites occurred only within variants of a single strain, while fewer sites were present in at least one genotypic variant of two (eight of 42) or all three (four of 42) strains (Table 5).

Each of the 42 restriction endonuclease sites mapped and the deletion unique to genotype 14 were used as characters to produce a binary character state code for each genotype (data not shown). A phylogenetic analysis based on parsimony using the binary character state codes resulted in a majority-rule consensus tree based on 1,000 bootstrap replicates (data not shown). This phylogenetic analysis supported the grouping of 41 of 43 genotypes into three taxa (strains Worland, $\mathrm{CFH}$, and $\mathrm{Cal} / \mathrm{Logan}$ ) that were consistent with strain assignments presented in Table 4 and Figure 2. Only genotypes 37 and 38 remained unresolved in the analysis. The lack of resolution of genotypes 37 and 38 was likely the result of these genotypes sharing more sites in common (relative to other genotypes) with variants of both the Worland and CFH strains and of the limited number of characters used in the analysis. Nonetheless, both genotypes 37 and 38 were tentatively considered as variants of the Worland strain, since the sugar beet isolates from which these genotypes were derived expressed mild symptoms as do other Worland isolates, and since the regions of DNA containing cis- and trans-acting elements of strain-specific DNA replication $(6,7)$ had restriction endonuclease map profiles typical of other Worland genotypic variants.

\section{DISCUSSION}

The demonstration of genetic diversity resident in contemporary field populations of BCTV in the western United States provides much needed data on genotypic variability and population structure of this economically important and widespread viral pathogen. It is clear from this study that BCTV genotypes vary both

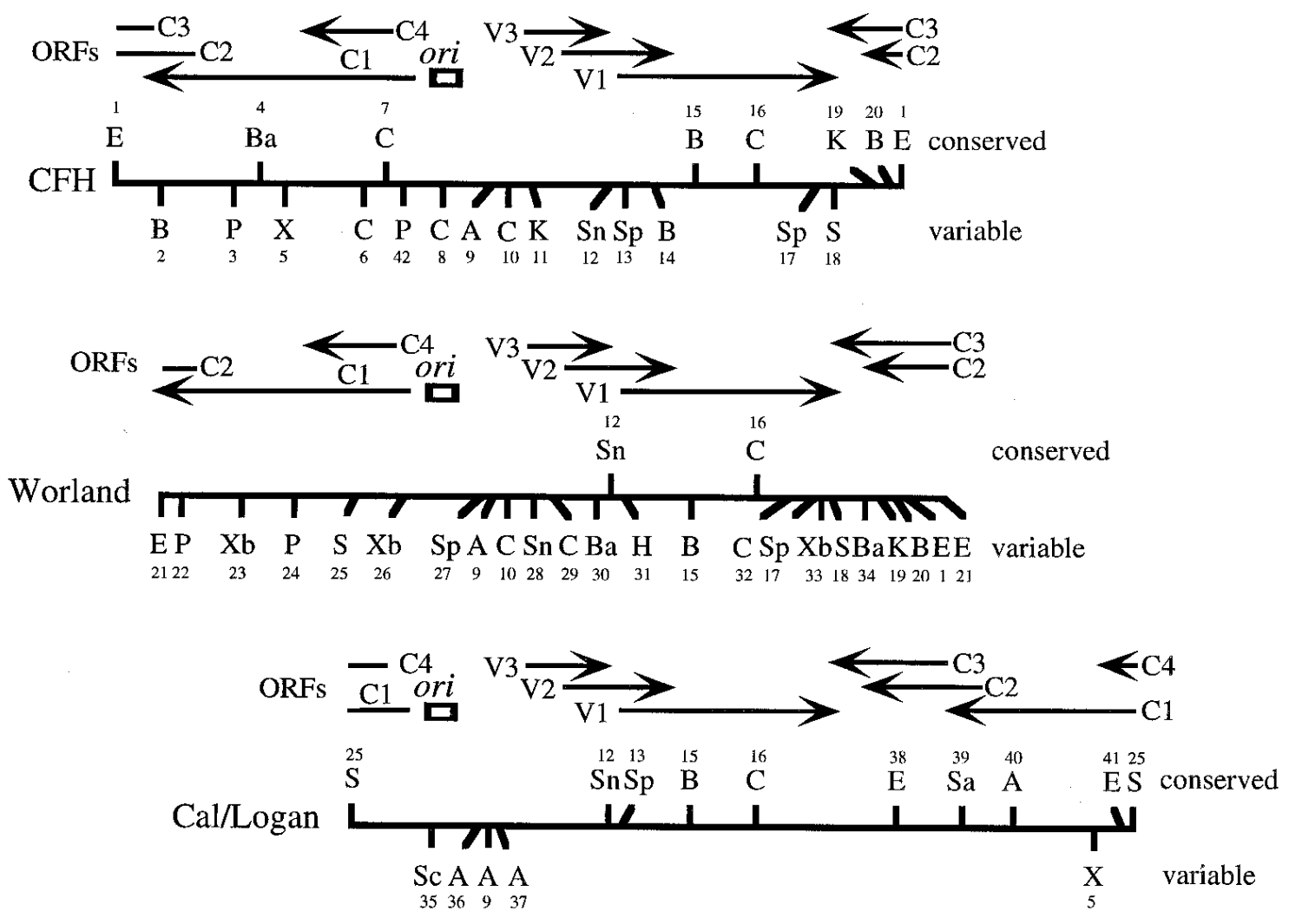

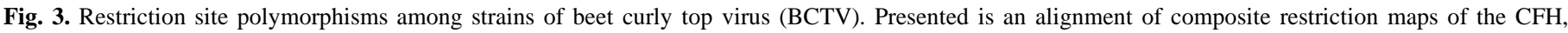

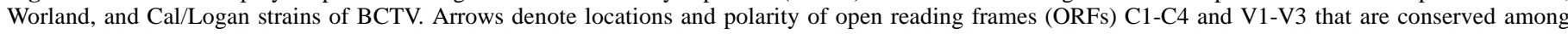

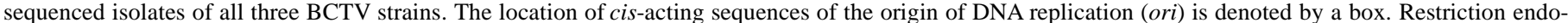

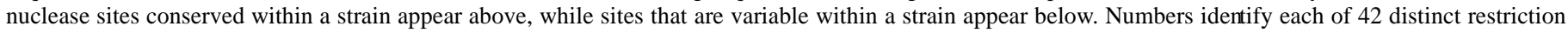

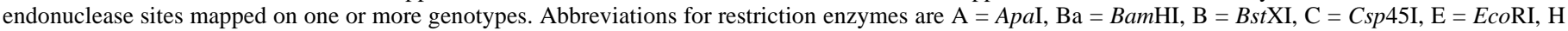
$=$ HindIII, K = KpnI, P = PvuII, Sa = SacI, S = SalI, Sc = ScaI, Sn = SnaBI, Sp = SpeI, Xb =XbaI, and X =XhoI. 
within and among strains. Furthermore, the phylogenetic analysis of BCTV genotypic variability supports the grouping of BCTV into the three currently recognized strains. Although the two genotypes (37 and 38) that were unresolved in the phylogenetic analysis could be viewed as variants of a fourth strain of BCTV, intermediate between the Worland and CFH strains, we argue for a more conservative interpretation in which these genotypes represent the most divergent examples of the Worland strain. How these two genotypes evolved is subject to speculation. One possibility is through interstrain recombination; another may be differential selection pressure(s) on specific regions of the genome. Complete sequencing of these two genomes may help to resolve this question.

Only two of the three recognized strains of BCTV were encountered in the field surveys conducted during 1994 and 1995. The Cal/Logan strain was not recovered from field samples. Given that Logan DNA sequences were used as a probe in all Southern and colony hybridizations, it seems unlikely that probe bias can account for the lack of recovery of $\mathrm{Cal} / \mathrm{Logan}$ clones from field samples. Furthermore, it seems unlikely that the selection and use of EcoRI to linearize RF DNA for cloning resulted in bias. Although all four known genotypic variants of the Cal/Logan strain contain two EcoRI sites (Fig. 2) and would not be recovered by gel purification in the size-selection strategy employed, there was no evidence for EcoRI fragments of the expected size (2.3 and 0.8 $\mathrm{kbp}$ ) by Southern hybridization. Previous analysis of nursery isolates dually infected with Cal/Logan and other BCTV strains (22) suggests that these two Cal/Logan-specific EcoRI fragments would not have gone undetected if present in the field samples.

The absence of the Cal/Logan strain in the current field survey raises several interesting questions. Perhaps the most important question may be whether the Cal/Logan strain is a significant factor in sugar beet production today. Although the results of this study indicated that the Cal/Logan strain was not associated with sugar beet cultivation in the localities surveyed, it may be premature to declare the $\mathrm{Cal} / \mathrm{Logan}$ strain a relic of the past. Although the $\mathrm{Cal} / \mathrm{Logan}$ strain may eventually be determined unimportant to contemporary sugar beet production, it remains quite possible that this current study was biased by failing to survey regions that still harbor this strain. Clearly, vast regions of the western United States have yet to be exhaustively sampled. In particular, no samples have been examined from Utah, Arizona, Nevada, Montana, and southern California. Utah would be a logical place to search for $\mathrm{Cal} /$ Logan variants, but the sugar beet industry has not operated on a commercial basis there for nearly 20 years.

As all but four isolates of BCTV examined were derived from infected sugar beets, it remains possible that our knowledge of BCTV genotypic diversity is skewed due to examination of isolates predominately from a single host. Currently, limited data concerning the genotypic diversity of BCTV in other hosts are available. Four isolates from Artesia, NM (Table 2), were obtained from pepper, and all four isolates yielded genotypic variants of the Worland strain. Interestingly, three sugar beet samples collected from Artesia, NM, during the same year contained genotypic variants of the $\mathrm{CFH}$ strain. Although these results are insufficient to determine potential effects of host species on BCTV population structure, they do point to the need to extend the current studies and examine the potential effects of other crop and weed host species on BCTV population structure.

The incidence of BCTV within a given region is known to vary from year to year, however, the variation in genotypic composition within a BCTV population over time has not been examined. In the 1994 survey conducted in the Texas panhandle (20), genotypic variants of the $\mathrm{CFH}$ strain were recovered. $\mathrm{CFH}$ also was the only BCTV strain detected in the limited survey of the same region conducted during 1995, yet the specific genotypic variants recovered in successive years were different (Table 2). Whether this difference reflects a change in the BCTV population structure from year to year or is an artifact of limited sampling remains unknown. Resolution of this question will require examination of larger numbers of BCTV isolates from the same region over a period of years.

Although genotypic variability among plant viruses is a universally accepted concept, very few studies have addressed this question in any depth. In addition to our study, several other reports in the literature suggest substantial genotypic variation in certain virus populations. Within the geminivirus family, examples of variability in field populations have been described for subgroup I viruses in Africa using restriction mapping, DNA sequence data, serological assays, and biological properties $(4,8,11$, 16). Polymerase chain reaction and DNA sequencing methods were used to assess variability of subgroup III bean golden mosaic virus isolates in the Dominican Republic (9). A recent survey of subgroup III geminiviruses in Mexico and the southern United States (25) further suggests that the current field situation with geminiviruses is complex and dynamic. Several studies on the pararetrovirus cauliflower mosaic virus $(1,12)$ document genotypic variability in a DNA virus that replicates through an RNA intermediate via reverse transcription. The quasispecies concept acknowledges that RNA viruses exist as a collection of genotypic variants, but detailed information on the extent of variability within and among plant RNA virus populations is limited. A recent report (15) on field populations of wheat streak virus demonstrated that methodologies required to assess RNA virus genotypic variability are now available. Clearly, additional studies employing molecular analyses to assess plant virus genotypic variability are required to collect sufficient data for application of quantitative population genetics models. It is anticipated that such studies will provide data to measure the influence of geographic isolation, gene flow, recombination, host and vector selection pressures, and time on the dynamics of plant virus population structure.

\section{ACKNOWLEDGMENTS}

This material is based upon work supported by the Beet Sugar Development Foundation and the Northern Illinois University Plant Molecular Biology Center. We thank J. R. Stander, D. Stallings, J. Gerik, G. Fisher, C. Rush, G. Heidel, and R. Larsen for providing beet curly top virus field isolates; and K. Ostrow, M. Friedman, and D. Hudspeth for providing technical support.

\section{LITERATURE CITED}

1. Al-Kaff, N., and Covey, S. N. 1994. Variation in biological properties of cauliflower mosaic virus clones. J. Gen. Virol. 75:3137-3145.

2. Bennett, C. W. 1971. The Curly Top Disease of Sugarbeet and Other Plants. Monograph 7. The American Phytopathological Society, St. Paul, $\mathrm{MN}$.

3. Briddon, R. W., Bedford, I. D., Tsai, J. H., and Markham, P. G. 1996. Analysis of the nucleotide sequence of the treehopper-transmitted geminivirus, tomato pseudo-curly top virus, suggests a recombinant origin. Virology 219:387-394.

4. Briddon, R. W., Lunness, P., Chamberlin, L. C. L., and Markham, P. G. 1994. Analysis of genetic variability of maize streak virus. Virus Genes 9:93-100.

5. Briddon, R. W., and Markham, P. G. 1995. Geminiviridae. Pages 158165 in: Virus Taxonomy. Report of the International Committee on Taxonomy of Viruses, 6th. F. A. Murphy, C. M. Fauquet, D. H. L. Bishop, A. W. Jarvis, G. P. Martelli, M. A. Mayo, and M. D. Summers, eds. Springer-Verlag, Vienna.

6. Choi, I.-R., and Stenger, D. C. 1995. Strain-specific determinants of beet curly top geminivirus DNA replication. Virology 206:904-912.

7. Choi, I.-R., and Stenger, D. C. 1996. The strain-specific cis-acting element of beet curly top virus geminivirus DNA replication maps to the directly repeated motif of the ori. Virology 226:122-126.

8. Dekker, E. L., Pinner, M. S., Markham, P. G., and van Regenmortel, M. H. V. 1988. Characterization of maize streak virus isolates from different plant species by polyclonal and monoclonal antibodies. J. Gen. Virol. 69:983-990.

9. Gilbertson, R. L., Rojas, M. R., Russell, D. R., and Maxwell, D. P. 1991. Use of asymmetric polymerase chain reaction and DNA sequencing to determine genetic variability of bean golden mosaic virus in the Domini- 
can Republic. J. Gen. Virol. 72:2843-2848.

10. Hormuzdi, S. G., and Bisaro, D. M. 1993. Genetic analysis of beet curly top virus: Evidence for three virion sense genes involved in movement and regulation of single- and double-stranded DNA levels. Virology 193: 900-909.

11. Hughes, F. L., Rybicki, E. P., and von Wechmar, M. B. 1992. Genome typing of southern African subgroup I geminiviruses. J. Gen. Virol. 73: 1031-1040.

12. Hull, R. 1980. Structure of the cauliflower mosaic virus genome III. Restriction endonuclease mapping of thirty-three isolates. Virology 100: 76-90.

13. Klute, K. A., Nadler, S. A., and Stenger, D. C. 1996. Horseradish curly top virus is a distinct subgroup II geminivirus species with rep and $C 4$ genes derived from a subgroup III ancestor. J. Gen. Virol. 77:1369-1378.

14. Lee, S., Stenger, D. C., Bisaro, D. M., and Davis, K. R. 1994. Identification of loci in Arabidopsis that confer resistance to geminivirus infection. Plant J. 6:525-535.

15. McNeil, J. E., French, R., Hein, G. L., Baenziger, P. S., and Eskridge, K. M. 1996. Characterization of genetic variability among natural populations of wheat streak virus. Phytopathology 86:1222-1227.

16. Mesfin, T., Bosque-Pérez, N. A., Buddenhagen, I. W., Thottappilly, G., and Olojede, S. O. 1992. Studies on maize streak virus isolates from grass and cereal hosts in Nigeria. Plant Dis. 76:789-795.

17. Stanley, J., Markham, P. G., Callis, R. J., and Pinner, M. S. 1986. The nucleotide sequence of an infectious clone of the geminivirus beet curly top virus. EMBO (Eur. Mol. Biol. Organ.) J. 5:1761-1767.

18. Stenger, D. C. 1994. Complete nucleotide sequence of the hypervirulent CFH strain of beet curly top virus. Mol. Plant-Microbe Interact. 7:154-157.

19. Stenger, D. C. 1994. Strain-specific mobilization and amplification of a transgenic defective-interfering DNA of the geminivirus beet curly top virus. Virology 203:397-402.

20. Stenger, D. C. 1995. Genotypic variability and the occurrence of less than genome-length viral DNA forms in a field population of beet curly top geminivirus. Phytopathology 85:1316-1322.

21. Stenger, D. C., Carbonaro, D., and Duffus, J. E. 1990. Genomic characterization of phenotypic variants of beet curly top virus. J. Gen. Virol. 71:2211-2215.

22. Stenger, D. C., Davis, K. R., and Bisaro, D. M. 1994. Recombinant beet curly top virus genomes exhibit both parental and novel pathogenic phenotypes. Virology 200:677-685.

23. Stenger, D. C., and Ostrow, K. M. 1996. Genetic complexity of a beet curly top virus population used to assess sugar beet cultivar response to infection. Phytopathology 86:929-933.

24. Stenger, D. C., Stevenson, M. C., Hormuzdi, S. G., and Bisaro, D. M. 1992. A number of subgenomic DNAs are produced following agroinoculation of plants with beet curly top virus. J. Gen. Virol. 73:237-242.

25. Torres-Pacheco, I., Garzón-Tiznado, J. A., Brown, J. K., Becarra-Flora A., and Rivera-Bustamante, R. F. 1996. Detection and distribution of geminiviruses in Mexico and the southern United States. Phytopathology 86:1186-1192. 